\title{
Measurements of electron and positron fluxes below the geomagnetic cutoff by the PAMELA magnetic spectrometer
}

Vladimir Mikhailov*1, O. Adriani ${ }^{2,3}$, G. C. Barbarino ${ }^{4,5}$, G. A. Bazilevskaya ${ }^{6}$, R. Bellotti ${ }^{7,8}$, M. Boezio ${ }^{9}$, E. A. Bogomolov ${ }^{10}$, M. Bongi ${ }^{11,3}$, V. Bonvicini ${ }^{9}$, S. Bottai ${ }^{3}$, A. Bruno ${ }^{7,8}$, F. Cafagna ${ }^{8}$, D. Campana ${ }^{5}$, P. Carlson ${ }^{12}$, M. Casolino ${ }^{13,14}$, G. Castellini $^{13}$, C. De Donato ${ }^{13}$, C. De Santis ${ }^{15}$, V. di Felice ${ }^{11,13}$, A. M. Galper ${ }^{1}$, A. V. Karelin ${ }^{1}$, S. V. Koldashov ${ }^{1}$, S. A. Koldobskiy ${ }^{1}$, S. Y. Krutkov ${ }^{10}$, A. N. Kvashnin ${ }^{6}$, A. A. Leonov ${ }^{1}$,V. V. Malakhov ${ }^{1}$, L. Marcelli ${ }^{15}$, M. Martucci ${ }^{15,16}$, A. G. Mayorov ${ }^{1}$, W. Menn ${ }^{17}$, M. Merge ${ }^{13,15}$, Y. V. Mikhailova ${ }^{1}$, E. Mocchiutti ${ }^{9}$, A. Monaco ${ }^{7,8}$, N. Mori $^{3}$, R. Munini ${ }^{9}$, G. Osteria ${ }^{5}$, B. Panico ${ }^{5}$ P. Papini ${ }^{3}$, M. Pearce ${ }^{12}$, P. Picozza ${ }^{13,15}$, M. Ricci ${ }^{16}$, S. B. Ricciarini ${ }^{18}$, M. Simon ${ }^{17}$, R. Sparvoli ${ }^{13,15}$, P. Spillantini ${ }^{2,3}$, Y. I. Stozhkov ${ }^{6}$, A. Vacchi ${ }^{9}, 19$, E. Vannuccini ${ }^{3}$, G. I. Vasilyev ${ }^{10}$, S. A. Voronov ${ }^{1}$, Y. T. Yurkin ${ }^{1}$, G. Zampa ${ }^{9}$, N. Zampa9

${ }^{1}$ National Research Nuclear University MEPhI, RU-115409 Moscow

${ }^{2}$ University of Florence, Department of Physics, I-50019 Sesto Fiorentino, Florence, Italy

${ }^{3}$ INFN, Sezione di Florence, I-50019 Sesto Fiorentino, Florence, Italy

${ }^{4}$ University of Naples “Federico II", Department of Physics, I-80126 Naples, Italy

${ }^{5}$ INFN, Sezione di Naples, I-80126 Naples, Italy

${ }^{6}$ Lebedev Physical Institute, RU-119991, Moscow, Russia

${ }^{7}$ University of Bari, Department of Physics, I-70126 Bari, Italy

${ }^{8}$ INFN, Sezione di Bari, I-70126 Bari, Italy

${ }^{9}$ INFN, Sezione di Trieste I-34149 Trieste, Italy

${ }^{10}$ Ioffe Physical Technical Institute, RU-194021 St. Petersburg, Russia

${ }^{11}$ Agenzia Spaziale Italiana (ASI) Science Data Center, I-00044 Frascati, Italy

${ }^{12}$ KTH and the Oskar Klein Centre, AlbaNova University Centre, SE-10691 Stockholm, Sweden

13 INFN, Sezione di Rome "Tor Vergata”, I-00133 Rome, Italy

${ }^{14}$ RIKEN, Advanced Science Institute, Wako-shi, Saitama, Japan

15 University of Rome “Tor Vergata”, Department of Physics, I-00133 Rome, Italy

${ }^{16}$ INFN, Laboratori Nazionali di Frascati, Via Enrico Fermi 40, I-00044 Frascati, Italy

${ }^{17}$ Universität Siegen, Department of Physics, D-57068 Siegen, Germany

18 IFAC, I-50019 Sesto Fiorentino, Florence, Italy

${ }^{19}$ University of Udine, Department of Mathematics and Informatics, I-33100 Udine, Italy E-mail: vvmikhajlov@mephi.ru 
We present a measurements of electron and positron fluxes below the geomagnetic cutoff rigidity in wide energy range from $50 \mathrm{MeV}$ to several GeVs by the PAMELA magnetic spectrometer. The instrument was launched on June 15th 2006 on-board the Resurs-DK satellite on low orbit with 70 degrees inclination and altitude between 350 and $600 \mathrm{~km}$. Features of spatial distributions of secondary electrons and positrons in the near Earth space, including the South Atlantic Anomaly, were investigated in terms of lifetime and geographical origin. The separation in stably trapped , long lifetime quasi-trapped, and short lifetime albedo components was performed on base of back tracing procedure in geomagnetic field. A significant difference in relative abundance of positrons with respect to electrons is seen for the stable trapped and the quasi-trapped populations what pointing out on differences in trapping mechanism of those populations.

35th International Cosmic Ray Conference - ICRC2017

10-20 July, 2017

Bexco, Busan, Korea

* Speaker. 


\section{Introduction}

Electrons and positrons produced by inelastic cosmic ray interactions with residual atmosphere execute their trajectories in the Earth magnetic field. Secondary electrons and positrons may perform many oscillations from one hemisphere to other and drift around the Earth in longitude direction. Flux of such secondary electrons was first calculated in paper [1]. This mechanism was confirmed in several experiments starting from seventieth. More modern Monte-Carlo simulation also were undertaken to explain results of AMS-01 experiments [2], e.g. [4, 3]. It was shown that well-known East -West effect leads to positron excess in near equatorial region for quasitrapped and trapped components from this source.

This work presents PAMELA spectrometer measurements of secondaries electrons and positrons under geomagnetic cut-off. The magnetic spectrometer PAMELA was launched on board the Resurs-DK1 satellite on the 15th of June 2006 and it was continuously taking data till 23 January 2016. The satellite had a quasi-polar $\left(70^{\circ}\right.$ inclination) elliptical orbit at an altitudes between 350 and $600 \mathrm{~km}$. After September 2010 the orbit was changed to circular with altitude $570 \mathrm{~km}$. Preliminary results of PAMELA observations of secondary electron and positron fluxes near the Earth, which were made in first years of the flight, were reported in papers [5, 7]. New approach based on analysis of particles trajectories in the Earth magnetosphere allows to separate stably trapped component of secondary electrons and positrons from quasitrapped (less then one drift around the Earth) and re-entrant albedo (only one oscillation between mirror points) components. Preliminary results pointed out on possible different origin of trapped and quasi-trapped species. In this work additional data set was added at low energy with improved data selection. Energy interval was enlarged till $70 \mathrm{MeV}$ and it allows to study secondary electrons and positrons generation and propagation in magnetosphere with more details.

\section{PAMELA spectrometer}

The PAMELA instrument consists of a Time-of-Flight system (TOF), an anticoincidence system (AC), a magnetic spectrometer, an electromagnetic imaging calorimeter, a shower tail catching scintillator and a neutron detector (ND) .

The TOF system gives the main trigger for particle acquisition, measures the value of the particle charge and its flight time while crossing the apparatus (the resolution is about $0.35 \mathrm{~ns}$ ). A rigidity is determined by the magnetic spectrometer, composed by a permanent magnet with a magnetic field intensity $0.4 \mathrm{~T}$ and a tracker with six micro-strip silicon planes.

The electron and positron identification is provided by the calorimeter, a series of X-Y strip silicon layers interleaved by 22 tungsten planes (16.3 radiation and 0.6 nuclear interaction lengths deep). Particles not cleanly entering the PAMELA acceptance are rejected by the anticoincidence system. Using of the TOF, the magnetic spectrometer, neutron detector and the calorimeter information allows identification of electrons and positrons and measuring their energy from $100 \mathrm{MeV}$ to several hundred $\mathrm{GeV}$ with high efficiency. The satellite had three axises stabilization and the PAMELA instrument was pointed mainly to a local zenith. The acceptance is about $21.6 \mathrm{~cm}^{2} \mathrm{sr}$ [6]. 


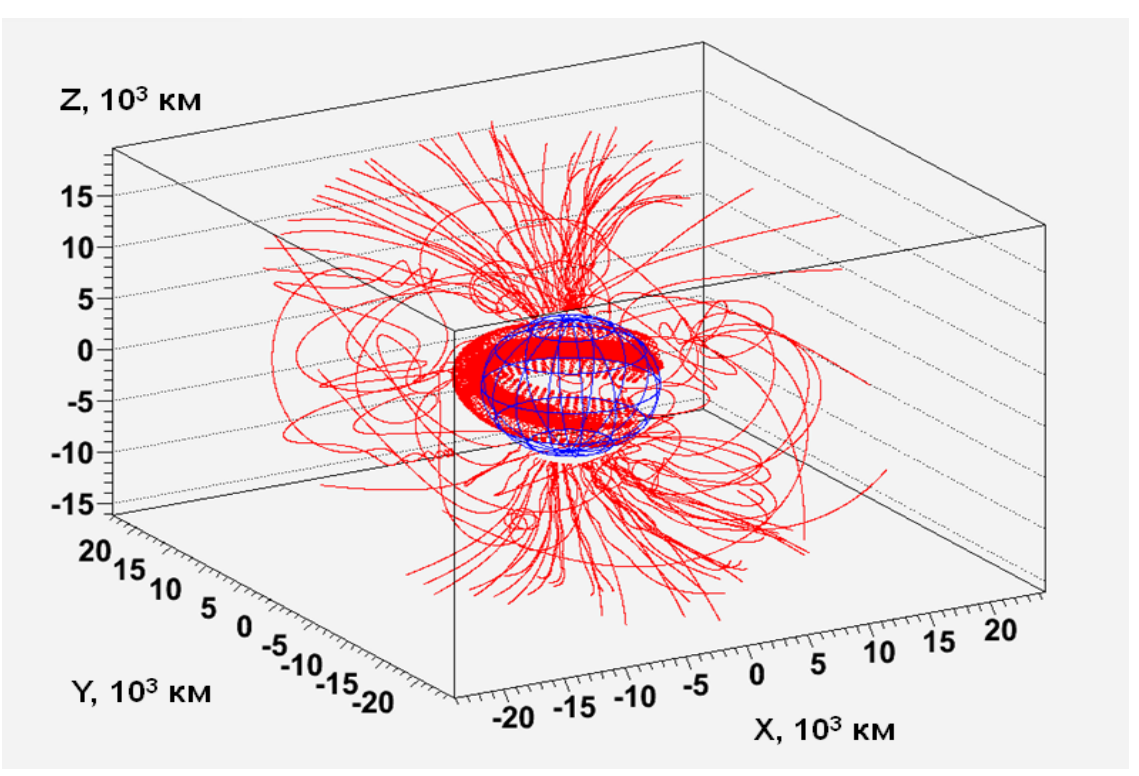

Figure 1: Examples calculated trajectories of electrons and positrons detected during several orbits

\section{Data Analysis}

Electrons and positrons were identified using information about $d E / d x$ energy losses in the spectrometer planes to determine charge $|Z|=1$, shower properties in the electromagnetic calorimeter, particle velocity $\beta \sim 1$ and a sign rigidity. The misidentification of protons and secondary pions is the main source of background. The rejection power based on combination the calorimeter data, rigidity, $\beta$ and ND data is about $\sim 10^{4}-10^{5}$ for protons and pions, keeping $\sim 70-80 \%$ efficiency of the electrons or positrons in energy range above $\sim 400 \mathrm{MeV}$. At low energy particles cross only few calorimeter planes and more strict cuts have to be applied to data from TOF, AC, ND and tracker.

Total accumulated statistic for electrons and positron is about $4 \times 10^{6}$ in whole energy range both for primary and secondary particles.

Using known geographical coordinates and orientation of the PAMELA instrument along the satellite orbit, the McIlvain geomagnetic coordinates L-shell and B were calculated for every event. For the calculation the IGRF model(http: //nssdcftp.gsfc.nasa.gov/models/geomagnetic/igrf) of the Earth magnetic field was used.

To determine origin of detected particles the backtracing procedure was applied for every identified events to obtain their trajectories in the Earth magnetosphere before detection. The tracing was stopped if particles touched the Earth atmosphere on $40 \mathrm{~km}$ altitude or escaped magnetosphere. Escaping boundary was chosen to be $20000 \mathrm{~km}$. In present analysis maximal time of tracing was $50 \mathrm{~s}$ that corresponds to drift time of $70 \mathrm{MeV}$ electrons around the Earth. MSIS mode (https://ccmc.gsfc.nasa.gov)l was used to determine the thickness of the traversed matter in the atmosphere. Trajectory analysis allows to determine particle origin for every individual

event. Figure 1 shows examples calculated trajectories of electrons and positrons detected during several orbits. Totally about $\sim 2 \times 10^{6}$ events were backtraced. 
[a]

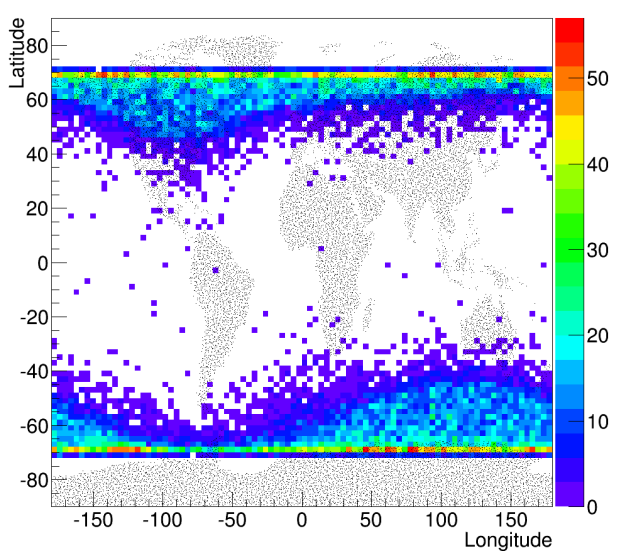

[b]

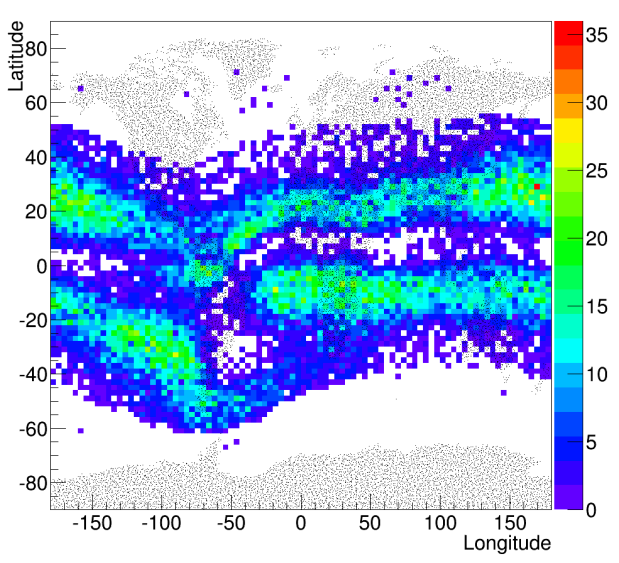

Figure 2: Regions where positrons were detected . Figure (a) shows primary particles. Figure (b) shows re-entrant albedo.

Typically galactic particles reach the satellite orbit for time less then 0.1 second from the boundary of the magnetosphere. But if electron or positron rigidity is just slightly lower geomagnetic cut-off rigidity this time may increase dramatically up to $\sim 1 \mathrm{~s}$ due to prolonged chaotic motions in the Earth magnetic field. Secondary re-entrant albedo have a peak near 0.1 second corresponding to particles motion along magnetic field lines from one hemisphere to the other. A long time intervals correspond to quasitrapped particles performing many oscillations. Drift time increases with decreasing of particles rigidity. There is alsofraction of particles with very long lifetime which make many drift motions around the Earth.

Figures 2 and 3 show geographical coordinates detected positrons of different origin. Primary particles are observed mainly in polar regions and above cut-off rigidity near equator (figure 2a). This component consist of mainly electrons. Secondary albedo component with small pitch-angles is observed below geomagnetic cut-off for all latitudes (figure $2 b$ ) and quasitrapped and trapped particles can be measured near equator and in South Atlantic Anomaly (SAA) (figure 3).

Maximum of count rate of trapped component is observed at L-shell about 1.18 and it is increasing with local magnetic field B decreasing below $0.215 \mathrm{G}$. Inside this region positron to electron ratio is maximal near West part of the South Atlantic Anomaly.

Finally, primary electrons and positrons with high rigidity $\mathrm{R}$ were excluded from analysis together with particles in transition region near geomagnetic cut-off, applying condition $R<10 / \mathrm{L}^{3}$ [GV], where L is geomagnetic L-shell.

\section{Results and Discussion}

Due to high accumulated statistic the experimental data show very detail dependence of electron and positron fluxes on particle energy in different spatial regions near the Earth. Figure 4 shows obtained in PAMELA experiment positron to electron $\mathrm{e}^{+} / \mathrm{e}^{-}$ratio for quasitrapped, reentrant and stably trapped particles. Results for albedo and quasitrapped particles are in agreement with AMS-01 data. Calculations in paper [4, 3] shows that well-known East -West effect leads 
[a]

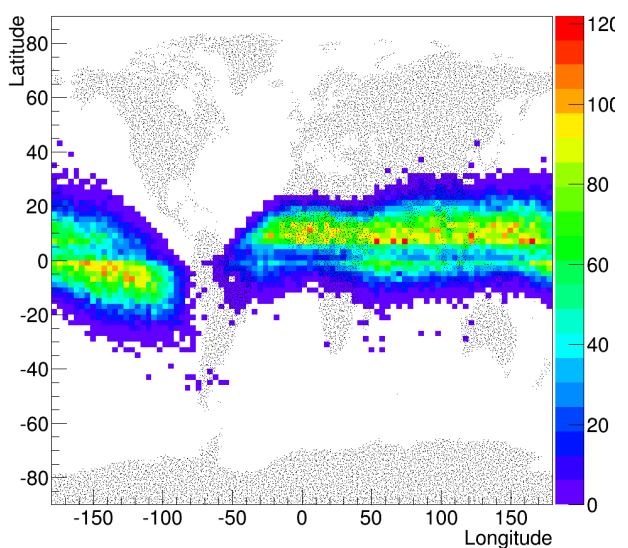

Figure 3: Regions where captured by the Earth magnetic field positrons were detected . Figure (a) shows quasitrapped particles. Figure (b) shows stably trapped particles.

to positron excess in near equatorial region for quasitrapped and trapped components from cosmic ray interaction with residual atmosphere. Surprisingly, positron fraction of trapped particles is much smaller then for quasitrapped component and more close to albedo one. This result also difficult to explained by additional productions of electrons and positrons inside radiation belt by trapping protons interactions. As it was calculated in paper [4] this mechanism produces mainly low energy $\mathrm{E}<100 \mathrm{MeV}$ positrons. Figure 5 presents count rates of trapped electrons and positrons in central part of SAA (right) where $\mathrm{B}<0.2 \mathrm{G}$ and near border of the region $0.2<\mathrm{B}<0.23 \mathrm{G}$ (left) at L-hell 1.18. With more deep entering into radiation belt positron fraction is decreasing and electrons begin to prevail.

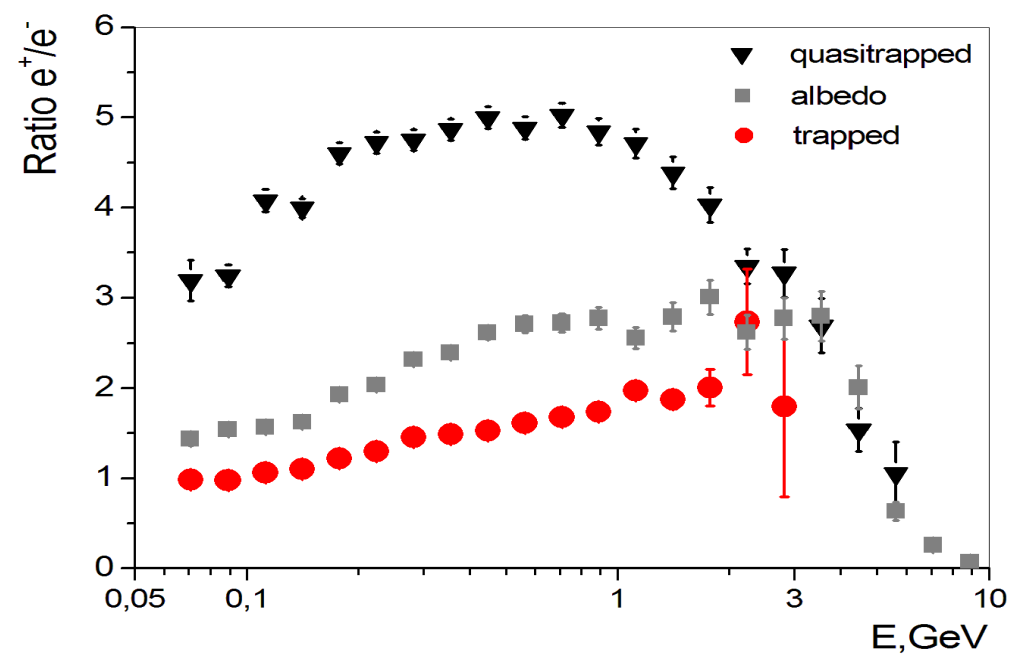

Figure 4: Positron to electron $\mathrm{e}^{+} / \mathrm{e}^{-}$ratio for quasitrapped, re-entrant and stably trapped particles. 


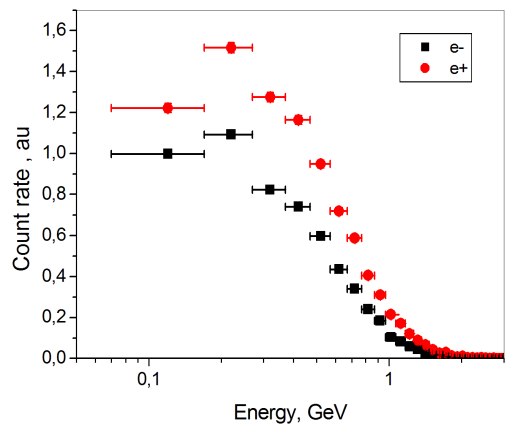

[a]

Figure 5: Trapped positron and electron count rate vs energy. Figure (a) shows region $B>0.2$ G. Figure (b) shows region $\mathrm{B}<0.2 \mathrm{G}$.

\section{Summary}

Secondary electron and positron fluxes have complex spatial structure caused by geomagnetic field, production cross-section and atmospheric absorption. Measured in the PAMELA experiment fluxes of electrons and positrons point out on differences in production or trapping mechanisms for stably trapped into inner radiation belts particles and quasitrapped particles on low edge of the belt.

\section{Acknowlegments}

We acknowledge support from the Italian Space Agency (ASI) under the program "Programma PAMELA - attivita' scientifica di analisi dati in fase E, Deutsches Zentrum fur Luft- und Raumfahrt (DLR), the Russian Ministry of Education and Science (project No.3.2131.2017), Russian Federal Space Agency, V. M. thanks for the support from National Research Nuclear University MEPhI in the framework of the Russian Academic Excellence Project (contract No. 02.a03.21.0005, 27.08.2013)

\section{References}

[1] N.L. Grigorov, Paper of AS USSR, 1977 (24) 810.

[2] J. Alcaraz, et al. [AMS01 collaboration] Phys. Lett. B, 2000 (484) 10.

[3] L. Derome, M. Buenerd, and Y. Liu, Phys. Lett. B, 2001 (515) 1.

[4] A.A. Gusev, U.B. Jayanthet, I.M. Martin et al., Journal of Geophys. Res. 2001 (106) 11 A26111

[5] O. Adriani et al.[PAMELA collaboration],Journal of Geophys. Res. 2009 A12218 doi:10.1029/2009JA014660

[6] P. Picozza, A.M. Galper, G. Castellini et al.[PAMELA collaboration], Astropart. Phys. 2007 (27) 296-315

[7] O.Adriani et al.[PAMELA collaboration],Phys.Rep. 2014 (544) 4 323-370 doi: 10.1016/j.physrep.2014.06.003 